\title{
The Study of Relationship between Neuroticism, Stressor and Stress Response
}

\author{
Xiaoyuan $\mathrm{Chu}^{1}$, Tianyi Duan ${ }^{2}$, Zhentao $\mathrm{Ma}^{3} \&{\mathrm{Yuan} \mathrm{Li}^{4}}^{4}$ \\ ${ }^{1}$ School of Economics and Resource Management, Beijing Normal University, Beijing, China \\ ${ }^{2}$ Institute of Educational Psychology and School Counseling, Beijing Normal University, Beijing, China \\ ${ }^{3}$ School of Government, Beijing Normal University, Beijing, China \\ ${ }^{4}$ Research Department, CIConsulting, Shenzhen, China \\ Correspondence: Xiaoyuan Chu, School of Economics and Resource Management, Beijing Normal University, \\ Beijing, China. Tel: 86-131-2675-6090. E-mail: seancxy@126.com
}

Received: May 4, 2015

doi:10.5539/ijef.v7n8p114
Accepted: May 21, 2015

URL: http://dx.doi.org/10.5539/ijef.v7n8p114

\begin{abstract}
Stress is everywhere in modern life. Increasingly more problems are emerging because of stress, which cause lots of economic losses. Based on the theoretical analysis, with first-hand data collection and using multiple regression models, this study explored the relationship between neuroticism, stressor and stress response and figured out interactive effect of neuroticism and stressor on stress response. We draw on the following conclusions: (1) The interaction term of stressor (work) and neuroticism as well as three stressors (health, family and social) are predictors of physiological stress response; (2) The interaction term of stressor (work) and neuroticism, two stressors (health and social) are predictors of psychological stress response; (3) The interaction term of stressor (health) and neuroticism as well as two stressors (social and family) are predictors of behavioral stress response; (4) The interaction term of stressor (health) and neuroticism, as well as three stressors (social, family and work) are predictors of total stress response.
\end{abstract}

Keywords: neuroticism, stressor, stress response, interactive effect

\section{Introduction}

Neuroticism reflects individual's emotional reaction tendency towards negative events and stimulus such as threat, setbacks or losses (Lahey, 2009). Neuroticism is usually defined as irritability, being easy to get angry, sad, anxious, worried, hostile, self-conscious and vulnerable (Weiss \& Costa, 2005). It reflects individual differences on emotional stability, and it is a continuum from emotional stability to instability (Verbeke \& Kenhove, 2002). Personality theorists believe that individuals with high level of neuroticism often experience more negative emotions, such as anxiety, worry, being sentimental or nervous. Individuals with low level of neuroticism, on the other hand, have slight and slow emotional reactions, and they are easier to restore calm. They are usually far more firm, steady and calm (Pevin, 2001). Behavioral studies also confirm a high correlation between negative emotions and neuroticism (DeNeve \& Cooper, 1998; Miller, Vachon, \& Lynam, 2009). High level of neuroticism will lead to more negative emotions. Some research found that high neuroticism can predict negative emotions in life: people with high level of neuroticism have more worries although they have better understanding ability. They have more negative emotions (Costa \& McCrae, 1980). Neuroticism and negative emotions such as fear, sadness, guilt, hostility, etc are strongly correlated (Watson \& Clark, 1992). Neuroticism can predict the degree of sadness as well as the enhancement of sad feelings (Gross, Sutton, \& Ketelaar, 1998). People of high neuroticism are not good at regulating negative emotions, and they tend to give more attention to situation which stimulates negative emotions and vent more negative emotions (Ng \& Diener, 2009). Neurotic individuals prone to process negative emotion information: they are more sensitive to threat-related stimuli (Osorio, Cohen, Escobar, Bartlett, \& Compton, 2003), and they have stronger responses to negative events in life (Gross, Sutton \& Ketelaar, 1998), and they tend to ruminate more on negative events (Muris, Roelofs, Rassin, Franken, \& Mayer, 2005); there are more negative emotions in their cognitive schema, and they tend to extract negative perceptions (Robinson, Ode, Moeller, \& Goetz, 2007). There are certain mechanisms between neuroticism and negative emotions system (Costa \& McCrae, 1980; Meyer \& Shack, 1989). In the face of aversive stimuli, the 
skin temperature of high neuroticism group rises more compared with low neuroticism group. While regulating the negative emotions, the skin temperature of high neuroticism group rises more slowly (Huang \& Guo, 2003). Neuroticism and stress responses are significantly and positively correlated. Individuals of high neuroticism react more intensely to external stimuli than average people, and they are poorer in regulating and coping with their own emotions. They tend to be in a bad mood and are weaker in thinking, decision making and responding to external pressures effectively. As a result, they are more likely to experience feeling of danger, threats and to feel nervous, fear, worried and anxious when facing stress. These emotional reactions can be reflected in their physiological, psychological and behavioral aspects (Chu, 2015).

On the basis of previous studies, this study attempts to use primary and secondary school teachers as subjects, investigating the relationship among neuroticism, stressor and stress responses, trying to discover the underlining mechanisms.

In order to reveal the relationship between neuroticism, stressors and stress responses, this paper constructed a basic model as follows:

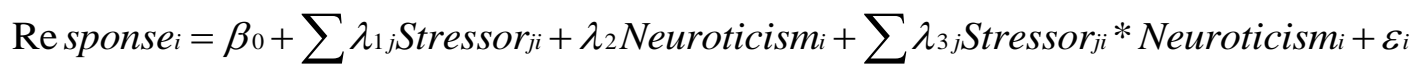

In the formula, $\mathrm{i}$ represents the subjects, $\mathrm{j}$ represents the type of stressors, Response represents the stress response (including four models: the total stress response, physiological stress responses, psychological stress responses and behavioral stress response), Stressor represents pressure source (including work stress, health stress, family stress and social stress), Stressor $_{\mathrm{ji}}{ }^{*}$ Neuroticism $_{\mathrm{i}}$ is the interaction term, and $\varepsilon_{\mathrm{i}}$ is the error term.

\section{Method}

\subsection{Subjects}

460 primary and secondary school teachers were recruited as subjects and 432 questionnaires were returned. After excluding invalid questionnaires, we finally obtained 428 valid questionnaires. The valid response rate is 93.04\%. The basic information of the sample is in Table 1 as below.

\subsection{Research Instrument}

\subsubsection{Neuroticism}

Big Five Personality Inventory, namely NEO-Personality Inventory is used to measure neuroticism. This scale is based on the Big Five personality theory and was compiled by the American psychologist Costa Costa and McCrae McRae in 1987. After many years of use and revise, this scale has been of high reliability and validity. The scale uses five scoring system, whose range of scores is 1 to 5 points. Scoring higher or lower indicates some more obvious characteristics in neuroticism.

\subsubsection{Stressor and Stress Response}

Work Stress Scale for Primary and Secondary School Teacher was used to measure stressor and stress response in this study. The scale consists of two parts. The first part is the source of stress, including a total of 36 items in four dimensions. The four dimensions are: work stress, health stress, family stress and social stress. The second part is the stress response, including a total of 17 items in three dimensions. The three dimensions are physiological stress response, psychological stress response and behavioral stress responses. It has been testified that the liability and validity of the scale are good. Specifically, the scale uses five scoring system, whose range of scores is 0 to 4 points. Scoring higher or lower indicates some more obvious characteristics in certain aspects.

\subsection{Research Process}

The questionnaires were administrated with the unified instructions. And the questionnaires, with no time limitation, were collected on the spot and checked one by one with invalid ones eliminated. This research employed SPSS19.0 for statistical analysis, which includes analysis of variance, correlation analysis and analysis of regression. 
Table 1. Basic information of the sample and the F-test for the stress response

\begin{tabular}{|c|c|c|c|c|c|c|c|c|}
\hline $\begin{array}{c}\text { Demographic } \\
\text { Variable }\end{array}$ & & $\mathrm{N}$ & Percentage & $\begin{array}{l}\text { Statistical } \\
\text { value }\end{array}$ & $\begin{array}{c}\text { Physiological } \\
\text { Stress } \\
\text { Response }\end{array}$ & $\begin{array}{c}\text { Psychological } \\
\text { Stress } \\
\text { Response }\end{array}$ & $\begin{array}{c}\text { Behavioral } \\
\text { Stress } \\
\text { Response }\end{array}$ & $\begin{array}{l}\text { Total Stress } \\
\text { Response }\end{array}$ \\
\hline \multirow{5}{*}{ Marital status } & Unmarried & 85 & 20.4 & & $8.22 \pm 3.704$ & $11.81 \pm 6.089$ & $2.67 \pm 1.572$ & $21.91 \pm 9.446$ \\
\hline & Married & 323 & 77.5 & & $8.77 \pm 3.777$ & $14.6 \pm 6.762$ & $4.17 \pm 2.517$ & $27.4 \pm 11.295$ \\
\hline & Divorced & 9 & 2.2 & & $10.25 \pm 3.732$ & $18.38 \pm 5.605$ & $4.6 \pm 2.608$ & $38.25 \pm 12.997$ \\
\hline & & & & $\mathrm{F}$ & 1.41 & 7.636 & 9.685 & 8.104 \\
\hline & & & & $\mathrm{P}$ & 0.245 & 0.001 & 0.000 & 0.000 \\
\hline \multirow{5}{*}{$\begin{array}{l}\text { Types of } \\
\text { School }\end{array}$} & Elementary & 154 & 38.1 & & $10.16 \pm 3.733$ & $15.25 \pm 7.170$ & $4.23 \pm 2.519$ & $29.24 \pm 12.596$ \\
\hline & Junior High & 77 & 19.1 & & $7.89 \pm 3.486$ & $13.29 \pm 6.823$ & $3.35 \pm 2.077$ & $25.19 \pm 10.487$ \\
\hline & Senior High & 173 & 42.8 & & $7.66 \pm 3.423$ & $13.33 \pm 6.283$ & $3.89 \pm 2.508$ & $24.92 \pm 10.251$ \\
\hline & & & & $\mathrm{F}$ & 22.082 & 3.863 & 2.87 & 5.18 \\
\hline & & & & $\mathrm{P}$ & 0.000 & 0.022 & 0.058 & 0.006 \\
\hline \multirow{6}{*}{ Service Year } & $\leq 5$ & 71 & 17 & & $8.07 \pm 3.969$ & $12.32 \pm 5.947$ & $2.96 \pm 2.345$ & $23.11 \pm 10.484$ \\
\hline & $5<\leq 10$ & 146 & 35 & & $8.21 \pm 3.472$ & $13.81 \pm 6.470$ & $3.65 \pm 2.182$ & $25.62 \pm 10.093$ \\
\hline & $10<\leq 20$ & 144 & 34.5 & & $9.01 \pm 4.022$ & $15.44 \pm 7.266$ & $4.59 \pm 2.443$ & $28.93 \pm 12.41$ \\
\hline & $>20$ & 56 & 13.4 & & $9.88 \pm 3.390$ & $13.87 \pm 6.885$ & $3.85 \pm 2.912$ & $26.32 \pm 11.267$ \\
\hline & & & & $\mathrm{F}$ & 3.636 & 3.643 & 6.246 & 3.536 \\
\hline & & & & $\mathrm{P}$ & 0.013 & 0.013 & 0.000 & 0.015 \\
\hline \multirow{4}{*}{ Child(ren) } & With & 236 & 66.5 & & $8.86 \pm 3.81$ & $14.55 \pm 6.911$ & $4.44 \pm 2.684$ & $27.73 \pm 11.958$ \\
\hline & Without & 119 & 33.5 & & $8.14 \pm 3.379$ & $13.15 \pm 6.085$ & $2.90 \pm 1.429$ & $24.14 \pm 9.408$ \\
\hline & & & & $\mathrm{F}$ & 3.063 & 3.405 & 33.587 & 7.664 \\
\hline & & & & $\mathrm{P}$ & 0.081 & 0.066 & 0.000 & 0.006 \\
\hline \multirow{4}{*}{ Gender } & Male & 97 & 23.4 & & $8.19 \pm 3.756$ & $13.69 \pm 6.629$ & $4.40 \pm 3.007$ & $26.24 \pm 10.444$ \\
\hline & Female & 317 & 76.6 & & $8.81 \pm 3.78$ & $14.22 \pm 6.844$ & $3.77 \pm 2.232$ & $26.63 \pm 11.562$ \\
\hline & & & & $\mathrm{F}$ & 2.01 & 0.427 & 4.093 & 0.074 \\
\hline & & & & $P$ & 0.157 & 0.514 & 0.044 & 0.786 \\
\hline
\end{tabular}

\section{Results}

\subsection{Correlation Analysis of Neuroticism, Stressors and Stress Response}

A correlation analysis of the stressor (as well as its dimensions) and the total stress response (as well as its dimensions) was conducted. As shown in Table 2, all the dimensions of stressors are significantly positively correlated with stress response and its dimensions. And the correlation analysis of neuroticism trait and stress response found that neuroticism is positively correlated with the total stress response and all its dimensions.

Table 2. Correlation matrix of variables

\begin{tabular}{|c|c|c|c|c|c|c|c|c|c|}
\hline Variable & 1 & 2 & 3 & 4 & 5 & 6 & 7 & 8 & 9 \\
\hline 1 Neuroticism & 1 & & & & & & & & \\
\hline 2 Stressor (work) & $.275^{* *}$ & 1 & & & & & & & \\
\hline 3 Stressor (health) & $.340 * *$ & $.643 * *$ & 1 & & & & & & \\
\hline 4 Stressor (family) & .055 & $.457 * *$ & $.285^{* *}$ & 1 & & & & & \\
\hline 5 Stressor (social) & $.127 * *$ & $.591 * *$ & $.337 * *$ & $.418 * *$ & 1 & & & & \\
\hline 6 Physiological Stress Response & $.247 * *$ & $.476^{* *}$ & $.447 * *$ & $.352 * *$ & $.382 * *$ & 1 & & & \\
\hline 7 Psychological Stress Response & $.327 * *$ & $.579 * *$ & $.536^{* * *}$ & $.357 * *$ & $.460 * *$ & $.665 * *$ & 1 & & \\
\hline 8 Behavioral Stress Response & $.249 * *$ & $.415^{* *}$ & $.483^{* *}$ & $.315^{* * *}$ & $.347 * *$ & $.539 * *$ & $.673 * *$ & 1 & \\
\hline 9 Total Stress Response & $.318 * *$ & $.568 * *$ & $.565^{* * *}$ & $.393 * *$ & $.460 * *$ & $.841 * *$ & $.908 * *$ & $.850 * *$ & 1 \\
\hline Minimum & 20 & .05 & 0 & 0 & 0 & 0 & 0 & 0 & .20 \\
\hline Maximum & 80 & 3.48 & 4.00 & 4.00 & 4.00 & 4.00 & 3.88 & 3.75 & 10.96 \\
\hline Mean & 48.62 & 1.83 & 1.70 & 1.67 & 2.13 & 1.73 & 1.78 & 1.30 & 4.82 \\
\hline
\end{tabular}




\subsection{Regression Analysis of Neuroticism, Stressors on Stress Response}

In order to understand the combined effect of stressors and neuroticism on the stress response, we take total stress response, physiological stress response, psychological stress response and behavioral stress response respectively as the predicted variable, and stressor (work), stressor (health), stressor (family), stressor (social), neuroticism and interaction terms of neuroticism and above-mentioned stressors as the predictive variables to do the regression analysis. The analysis results are shown in Tables 4-7.

As shown in Table 4, model 1 shows that all the four stressors are significant in the total stress response regression model; Model 2 indicates that in consideration of the stressors, the neuroticism is significant in the total stress response regression model; Model 3 indicates that in the total stress response regression model, neuroticism has a significant interactive effect with stressor (work); Model 4 indicates that in the total stress response regression model, neuroticism has a significant interactive effect with stressor (health); Model 5 and 6 indicate that in the total stress response regression model, neuroticism has no significant interactive effect with stressor (family) or stressor (social). To further figure out which part of stress response can be affected by the interaction between neuroticism and stressors, we did some more regression with physiological stress response, psychological stress response and behavioral stress response respectively as the predicted variable.

As shown in Table 5, model 1 indicates that stressor (family), stressor (health) and stressor (social) are significant predictors in the physiological stress response regression model; Model 2 indicates that in consideration of the stressor, the neuroticism is a significant predictors in the physiological stress response regression model; Model 3 to 7 indicate that in the physiological stress response regression model, neuroticism has no significant interaction with stressors.

As shown in Table 6, model 1 indicates that stressor (work), stressor (health) and stressor (social) are significant predictors in the psychological stress response regression model; Model 2 indicates that in consideration of the stressor, neuroticism is significant in the psychological stress response regression model; Model 3 to 7 indicate that in the psychological stress response regression model, neuroticism has no significant interaction with stressors.

As shown in Table 7, model 1 indicates that stressor (family), stressor (health) and stressor (social) are significant predictors in the behavioral stress response regression model; Model 2 indicates that in consideration of the stressor, neuroticism is significant in the behavioral stress response regression model; Model 3 indicates that in the behavioral stress response regression model, neuroticism has a significant interactive effect with stressor (work); Model 4 indicates that in the behavioral stress response regression model, neuroticism has a significant interactive effect with stressor (health); Model 5 and 6 show that in the behavioral stress response regression model, neuroticism has no significant interaction with stressor (family) or stressor (social).

Table 4. Neuroticism, stressors and total stress response (predicted variable: total stress response)

\begin{tabular}{|c|c|c|c|c|c|c|c|}
\hline Predictive variable & Model 1 & Model 2 & Model 3 & Model 4 & Model 5 & Model 6 & Model 7 \\
\hline Neuroticism & & $\begin{array}{l}.132 * * \\
(3.401)\end{array}$ & $\begin{array}{l}-.123 \\
(-.995)\end{array}$ & $\begin{array}{l}-.045 \\
(-.498)\end{array}$ & $\begin{array}{l}.291 * * * \\
(3.778)\end{array}$ & $\begin{array}{l}.144 \\
(1.407)\end{array}$ & $\begin{array}{l}-.057 \\
(-.491)\end{array}$ \\
\hline Stressor (work) & $\begin{array}{l}.133 * \\
(2.311)\end{array}$ & $\begin{array}{l}.115^{*} \\
(2.012)\end{array}$ & $\begin{array}{l}.105 \\
(.618)\end{array}$ & & & & $\begin{array}{l}-.203 \\
(-.811)\end{array}$ \\
\hline Stressor (health) & $\begin{array}{l}.369 * * * \\
(7.714)\end{array}$ & $\begin{array}{l}.333^{* * *} \\
(6.884)\end{array}$ & & $.148(.835)$ & & & $\begin{array}{l}.141 \\
(.656)\end{array}$ \\
\hline Stressor (family) & $\begin{array}{l}.129 * * \\
(3.017)\end{array}$ & $\begin{array}{l}.143^{* *} \\
(3.366)\end{array}$ & & & $\begin{array}{l}.393^{*} \\
(2.260)\end{array}$ & & $\begin{array}{l}.197 \\
(1.212)\end{array}$ \\
\hline Stressor (social) & $\begin{array}{l}.220^{* * * *} \\
(4.709)\end{array}$ & $\begin{array}{l}.222 * * * * \\
(4.809)\end{array}$ & & & & $\begin{array}{l}.250 \\
(1.399)\end{array}$ & $\begin{array}{l}.423^{*} \\
(2.088)\end{array}$ \\
\hline Neuroticism*Stressor (work) & & & $\begin{array}{l}.570^{*} \\
(2.499)\end{array}$ & & & & $\begin{array}{l}.462 \\
(1.359)\end{array}$ \\
\hline Neuroticism*Stressor (health) & & & & $\begin{array}{l}.469^{*} \\
(2.169)\end{array}$ & & & $\begin{array}{l}.239 \\
(.912)\end{array}$ \\
\hline Neuroticism*Stressor (family) & & & & & $\begin{array}{l}-.010 \\
(-.055)\end{array}$ & & $\begin{array}{l}-.063 \\
(-.360)\end{array}$ \\
\hline Neuroticism*Stressor (social) & & & & & & $\begin{array}{l}.239 \\
(1.144)\end{array}$ & $\begin{array}{l}-.260 \\
(-1.086)\end{array}$ \\
\hline
\end{tabular}




\begin{tabular}{llllllll}
\hline Control variable & & & & & & & \\
Gender & $.085^{*}$ & $.079^{*}$ & .044 & .063 & $.114^{* *}$ & $.089^{*}$ & $.082^{*}$ \\
& $(2.256)$ & $(2.124)$ & $(1.082)$ & $(1.565)$ & $(2.607)$ & $(2.136)$ & $(2.203)$ \\
Age & $.151^{* * *}$ & $.141^{* * *}$ & $.125^{* *}$ & $.131^{* *}$ & .079 & $.180^{* * *}$ & $.140^{* * *}$ \\
& $(3.990)$ & $(3.763)$ & $(3.134)$ & $(3.255)$ & $(1.822)$ & $(4.252)$ & $(3.728)$ \\
$\mathrm{R} 2$ & .459 & .474 & .369 & .363 & .259 & .311 & .482 \\
Adjusted R2 & .451 & .465 & .362 & .356 & .250 & .303 & .468 \\
$\mathrm{~N}$ & 412 & 412 & 412 & 412 & 412 & 412 & 412 \\
\hline
\end{tabular}

Note. $* * *, * *$ and $*$ indicate that the coefficient is significant at the $0.01,0.05$ and 0.1 levels, respectively the same below.

Table 5. Neuroticism, stressor and physiological stress response (predicted variable: physiological stress response)

\begin{tabular}{|c|c|c|c|c|c|c|c|}
\hline Predictive variable & Model 1 & Model 2 & Model 3 & Model 4 & Model 5 & Model 6 & Model 7 \\
\hline Neuroticism & & $\begin{array}{l}.103^{*} \\
(2.351)\end{array}$ & $\begin{array}{l}-.041 \\
(-.303)\end{array}$ & $\begin{array}{l}.055 \\
(.551)\end{array}$ & $\begin{array}{l}.255^{* * *} \\
(3.174)\end{array}$ & $\begin{array}{l}.215^{*} \\
(1.974)\end{array}$ & $\begin{array}{l}.060 \\
(.455)\end{array}$ \\
\hline Stressor (work) & $\begin{array}{l}.124 \\
(1.913)\end{array}$ & $\begin{array}{l}.110 \\
(1.696)\end{array}$ & $\begin{array}{l}.197 \\
(1.067)\end{array}$ & & & & $\begin{array}{l}-.385 \\
(-1.358)\end{array}$ \\
\hline Stressor (health) & $\begin{array}{l}.267 * * * * \\
(4.958)\end{array}$ & $\begin{array}{l}.239 * * * \\
(4.352)\end{array}$ & & $\begin{array}{l}.315 \\
(1.615)\end{array}$ & & & $\begin{array}{l}.372 \\
(1.520)\end{array}$ \\
\hline Stressor (family) & $\begin{array}{l}.136^{* *} \\
(2.820)\end{array}$ & $\begin{array}{l}.147 * * \\
(3.046)\end{array}$ & & & $\begin{array}{l}.424 * \\
(2.336)\end{array}$ & & $\begin{array}{l}.206 \\
(1.114)\end{array}$ \\
\hline Stressor (social) & $\begin{array}{l}.177 * * \\
(3.367)\end{array}$ & $\begin{array}{l}.178 * * \\
(3.413)\end{array}$ & & & & $\begin{array}{l}.416^{*} \\
(2.188)\end{array}$ & $\begin{array}{l}.602 * * \\
(2.615)\end{array}$ \\
\hline Neuroticism*Stressor (work) & & & $\begin{array}{l}.323 \\
(1.302)\end{array}$ & & & & $\begin{array}{l}.697 \\
(1.808)\end{array}$ \\
\hline $\begin{array}{l}\text { Neuroticism*Stressor } \\
\text { (health) }\end{array}$ & & & & $\begin{array}{l}.120 \\
(.507)\end{array}$ & & & $\begin{array}{l}-.160 \\
(-.536)\end{array}$ \\
\hline $\begin{array}{l}\text { Neuroticism*Stressor } \\
\text { (family) }\end{array}$ & & & & & $\begin{array}{l}-.093 \\
(-.476)\end{array}$ & & $\begin{array}{l}-.074 \\
(-.372)\end{array}$ \\
\hline Neuroticism*Stressor (social) & & & & & & $\begin{array}{l}-.050 \\
(-.224)\end{array}$ & $\begin{array}{l}-.519 \\
(-1.905)\end{array}$ \\
\hline Control variable & & & & & & & \\
\hline Gender & $\begin{array}{l}.083 \\
(1.959)\end{array}$ & $\begin{array}{l}.078 \\
(1.858)\end{array}$ & $\begin{array}{l}.045 \\
(1.026)\end{array}$ & $\begin{array}{l}.064 \\
(1.444)\end{array}$ & $\begin{array}{l}.107^{*} \\
(2.347)\end{array}$ & $\begin{array}{l}.085 \\
(1.913)\end{array}$ & $\begin{array}{l}.086^{*} \\
(2.020)\end{array}$ \\
\hline Age & $\begin{array}{l}.150 * * * \\
(3.519)\end{array}$ & $\begin{array}{l}.142 * * \\
(3.344)\end{array}$ & $\begin{array}{l}.133 * * \\
(3.052)\end{array}$ & $\begin{array}{l}.135 * * \\
(3.044)\end{array}$ & $\begin{array}{l}.092 * \\
(2.031)\end{array}$ & $\begin{array}{l}.176^{* * * *} \\
(3.909)\end{array}$ & $\begin{array}{l}.136^{* *} \\
(3.204)\end{array}$ \\
\hline $\mathrm{R} 2$ & .315 & .324 & .253 & .230 & .193 & .216 & .332 \\
\hline Adjusted R2 & .304 & .312 & .244 & .220 & .183 & .206 & .314 \\
\hline $\mathrm{N}$ & 412 & 412 & 412 & 412 & 412 & 412 & 412 \\
\hline
\end{tabular}

Table 6. Neuroticism, stressor and psychological stress response (predicted variable: psychological stress response)

\begin{tabular}{llllllll}
\hline Predictive variable & Model 1 & Model 2 & Model 3 & Model 4 & Model 5 & Model 6 & Model 7 \\
\hline Neuroticism & & $.135^{* *}$ & .022 & .007 & $.314 * * *$ & .154 & .050 \\
& & $(3.405)$ & $(.175)$ & $(.080)$ & $(3.963)$ & $(1.490)$ & $(.422)$ \\
Stressor (work) & $.221^{* * *}$ & $.202^{* *}$ & .323 & & & .252 \\
& $(3.728)$ & $(3.442)$ & $(1.906)$ & & & $(.977)$ \\
Stressor (health) & $.315^{* * *}$ & $.278^{* * *}$ & & .208 & & -.033 \\
& $(6.408)$ & $(5.591)$ & & $(1.142)$ & & $(-.149)$ \\
Stressor (family) & .060 & .074 & & & $.389 *$ & .148 \\
Stressor (social) & $(1.368)$ & $(1.704)$ & & & $(2.171)$ & .252 & $(.881)$ \\
& $.211^{* * *}$ & $.213 * * *$ & & & $(1.398)$ & $(.999)$ \\
Neuroticism*Stressor (work) & $(4.385)$ & $(4.482)$ & & & & -.053 \\
& & & .288 & & & $(-.152)$ \\
\hline
\end{tabular}




\begin{tabular}{|c|c|c|c|c|c|c|c|}
\hline Neuroticism*Stressor (health) & & & & $\begin{array}{l}.363 \\
(1.634)\end{array}$ & & & $\begin{array}{l}.387 \\
(1.428)\end{array}$ \\
\hline Neuroticism*Stressor (family) & & & & & $\begin{array}{l}-.058 \\
(-.301)\end{array}$ & & $\begin{array}{l}-.078 \\
(-.435)\end{array}$ \\
\hline Neuroticism*Stressor (social) & & & & & & $\begin{array}{l}.230 \\
(1.086)\end{array}$ & $\begin{array}{l}-.011 \\
(-.045)\end{array}$ \\
\hline \multicolumn{8}{|l|}{ Control variable } \\
\hline Gender & $\begin{array}{l}.029 \\
(.758)\end{array}$ & $\begin{array}{l}.023 \\
(.608)\end{array}$ & $\begin{array}{l}-.004 \\
(-.111)\end{array}$ & $\begin{array}{l}.020 \\
(.483)\end{array}$ & $\begin{array}{l}.065 \\
(1.457)\end{array}$ & $\begin{array}{l}.045 \\
(1.072)\end{array}$ & $\begin{array}{l}.021 \\
(.547)\end{array}$ \\
\hline Age & $\begin{array}{l}.127 * * \\
(3.247)\end{array}$ & $\begin{array}{l}.116^{* *} \\
(3.013)\end{array}$ & $\begin{array}{l}.095^{*} \\
(2.365)\end{array}$ & $\begin{array}{l}.099 * \\
(2.395)\end{array}$ & $\begin{array}{l}.053 \\
(1.186)\end{array}$ & $\begin{array}{l}.148 * * \\
(3.471)\end{array}$ & $\begin{array}{l}.117 * * \\
(3.031)\end{array}$ \\
\hline R2 & .427 & .443 & .368 & .329 & .216 & .297 & .447 \\
\hline Adjusted R2 & .419 & .433 & .360 & .321 & .207 & .288 & .432 \\
\hline $\mathrm{N}$ & 412 & 412 & 412 & 412 & 412 & 412 & 412 \\
\hline
\end{tabular}

Table 7. Neuroticism, stressor and behavioral stress response (predicted variable: behavioral stress response)

\begin{tabular}{|c|c|c|c|c|c|c|c|}
\hline Predictive variable & Model 1 & Model 2 & Model 3 & Model 4 & Model 5 & Model 6 & Model 7 \\
\hline Neuroticism & & $\begin{array}{l}.102 * \\
(2.323)\end{array}$ & $\begin{array}{l}-311 * \\
(-2.284)\end{array}$ & $\begin{array}{l}-181 \\
(-1.871)\end{array}$ & $\begin{array}{l}.184 * \\
(2.272)\end{array}$ & $\begin{array}{l}.006 \\
(.056)\end{array}$ & $\begin{array}{l}-.264 * \\
(-2.023)\end{array}$ \\
\hline Stressor (work) & $\begin{array}{l}-.008 \\
(-.119)\end{array}$ & $\begin{array}{l}-.022 \\
(-.336)\end{array}$ & $\begin{array}{l}-.265 \\
(-1.418)\end{array}$ & & & & $\begin{array}{l}-.443 \\
(-1.575)\end{array}$ \\
\hline Stressor (health) & $\begin{array}{l}.379 * * * \\
(7.021)\end{array}$ & $\begin{array}{l}.351 * * * \\
(6.382)\end{array}$ & & $\begin{array}{l}-.138 \\
(-.727)\end{array}$ & & & $\begin{array}{l}.053 \\
(.219)\end{array}$ \\
\hline Stressor (family) & $\begin{array}{l}.146^{* *} \\
(3.036)\end{array}$ & $\begin{array}{l}.157 * * \\
(3.259)\end{array}$ & & & $\begin{array}{l}.211 \\
(1.155)\end{array}$ & & $\begin{array}{l}.163 \\
(.891)\end{array}$ \\
\hline Stressor (social) & $\begin{array}{l}.183^{* *} \\
(3.483)\end{array}$ & $\begin{array}{l}.185^{* * * *} \\
(3.529)\end{array}$ & & & & $\begin{array}{l}-.014 \\
(-.073)\end{array}$ & $\begin{array}{l}.315 \\
(1.380)\end{array}$ \\
\hline Neuroticism*Stressor (work) & & & $\begin{array}{l}.887 \text { *** } \\
(3.523)\end{array}$ & & & & $\begin{array}{l}.613 \\
(1.602)\end{array}$ \\
\hline Neuroticism*Stressor (health) & & & & $\begin{array}{l}.733^{* *} \\
(3.175)\end{array}$ & & & $\begin{array}{l}.366 \\
(1.237)\end{array}$ \\
\hline Neuroticism*Stressor (family) & & & & & $\begin{array}{l}.126 \\
(.641)\end{array}$ & & $\begin{array}{l}-.010 \\
(-.050)\end{array}$ \\
\hline Neuroticism*Stressor (social) & & & & & & $\begin{array}{l}.434 \\
(1.923)\end{array}$ & $\begin{array}{l}-.180 \\
(-.665)\end{array}$ \\
\hline Control variable & & & & & & & \\
\hline Gender & $\begin{array}{l}.114 * * \\
(2.678)\end{array}$ & $\begin{array}{l}.109 * \\
(2.581)\end{array}$ & $\begin{array}{l}.077 \\
(1.739)\end{array}$ & $\begin{array}{l}.084 \\
(1.952)\end{array}$ & $\begin{array}{l}.128 * * \\
(2.789)\end{array}$ & $\begin{array}{l}.106^{*} \\
(2.343)\end{array}$ & $\begin{array}{l}.113 * * \\
(2.682)\end{array}$ \\
\hline Age & $\begin{array}{l}.119^{* *} \\
(2.778)\end{array}$ & $\begin{array}{l}.111^{*} \\
(2.603)\end{array}$ & $\begin{array}{l}.101 * \\
(2.296)\end{array}$ & $\begin{array}{l}.110^{*} \\
(2.558)\end{array}$ & $\begin{array}{l}.064 \\
(1.394)\end{array}$ & $\begin{array}{l}.145^{* *} \\
(3.195)\end{array}$ & $\begin{array}{l}.111^{* *} \\
(2.634)\end{array}$ \\
\hline $\mathrm{R} 2$ & .312 & .321 & .231 & .273 & .182 & .201 & .341 \\
\hline Adjusted R2 & .302 & .310 & .221 & .264 & .172 & .191 & .323 \\
\hline $\mathrm{N}$ & 412 & 412 & 412 & 412 & 412 & 412 & 412 \\
\hline
\end{tabular}

To further reveal the interactive effect of neuroticism and stressor on stress responses, we conducted a stepwise regression analysis, with physiological stress response, psychological stress response, behavioral stress response and total stress response as predicted variable respectively, and stressor (work), stressor (health), stressor (family), stressor (social), neuroticism and the interaction terms between neuroticism and the above-mentioned stressors as predictive variables. The regression results are shown in Table 8. 
Table 8. Stepwise regression analysis of neuroticism and stressors on dimensions of stress response

\begin{tabular}{|c|c|c|c|c|c|}
\hline Predicted variable & Predictive variable & Standardized $\beta$ & $\mathrm{T}$ & $\mathrm{P}$ & Adjusted $\mathrm{R}^{2}$ \\
\hline \multirow[t]{5}{*}{ Physiological Stress Response } & Neuroticism*Stressor (work) & .195 & 3.375 & .001 & 0.319 \\
\hline & Stressor (family) & .131 & 2.814 & .005 & \\
\hline & Stressor (health) & .231 & 4.330 & .000 & \\
\hline & Stressor (social) & .167 & 3.366 & .001 & \\
\hline & Age & .131 & 3.109 & .002 & \\
\hline \multirow[t]{4}{*}{ Psychological Stress Response } & Neuroticism*Stressor (work) & .282 & 5.430 & .000 & 0.439 \\
\hline & Stressor (health) & .287 & 5.942 & .000 & \\
\hline & Stressor (social) & .238 & 5.562 & .000 & \\
\hline & Age & .124 & 3.290 & .001 & \\
\hline \multirow[t]{5}{*}{ Behavioral Stress Response } & Neuroticism*Stressor (health) & .400 & 9.252 & .000 & 0.301 \\
\hline & Stressor (family) & .163 & 3.563 & .000 & \\
\hline & Stressor (social) & .169 & 3.617 & .000 & \\
\hline & Age & .109 & 2.593 & .010 & \\
\hline & Gender & .104 & 2.518 & .012 & \\
\hline \multirow[t]{6}{*}{ Total Stress Response } & Neuroticism*Stressor (health) & .398 & 8.826 & .000 & 0.479 \\
\hline & Stressor (social) & .209 & 4.576 & .000 & \\
\hline & Stressor (family) & .147 & 3.504 & .001 & \\
\hline & Age & .142 & 3.817 & .000 & \\
\hline & Stressor (work) & .129 & 2.366 & .018 & \\
\hline & Gender & .077 & 2.071 & .039 & \\
\hline
\end{tabular}

The data in Table 8 indicates that:

The interaction term of stressor (work) and neuroticism, stressor (health), stressor (family) and stressor (social) entered into the regression equation on physiological stress response, with a predictive power of $31.9 \%$;

The interaction term of stressor (work) and neuroticism, stressor (health) and stressor (social) entered into the regression equation on psychological stress response, with a predictive power of $43.9 \%$;

The interaction term of stressor (health) and neuroticism, stressor (social) and stressor (family) entered into the regression equation on behavioral stress response, with a predictive power of $30.1 \%$;

The interaction term of stressor (health) and neuroticism, stressor (social), stressor (family) and stressor (work) entered into the regression equation on total stress response, with a predictive power of $47.9 \%$.

\section{Discussion}

Neuroticism reflects the individual's emotional adjustment process, mainly the tendency of individual to experience negative emotions and emotional instability. Individuals high in neuroticism exhibit obvious tendency of annoyance, insecurity and self-pity. They tend to be anxious, hostile, depressed, impulsive and fragile. Table 8 indicates the interaction term of stressor (work) and neuroticism can positively predict physiological stress response, which means that individuals high in neuroticism are more likely to be affected by work stress physiologically, while those low in neuroticism would be less likely to suffer from work stress physiologically. In another word, neuroticism is a risk factor for the physiological health in consideration of work stress and those who are more neurotic are more vulnerable to work stress physiologically. The interaction term of stressor (work) and neuroticism can positively predict psychological stress response, which means that individuals high in neuroticism are more likely to be affected by work stress psychologically, while those low in neuroticism would be less likely to suffer from work stress psychologically. In another word, neuroticism is a risk factor for the psychological health in consideration of work stress and those who are more neurotic are more vulnerable to work stress psychologically. The interaction term of stressor (health) and neuroticism can positively predict behavioral stress response, which means that individuals high in neuroticism are more likely to be affected by health stress behaviorally, while those low in neuroticism would be less likely to suffer from health stress behaviorally. In another word, neuroticism is a risk factor for the behavioral health in consideration of health stress and those who are more neurotic are more vulnerable to health stress behaviorally. Individuals of high neuroticism respond more intensely to external stressful stimulation than average people, and they are poorer in regulating and coping with their own emotions. They have relatively poor ability to adjust and cope with their own emotional states, which usually leads to bad mood. Their abilities of thinking, decision-making and dealing 
with stressor are relatively poor as well. So they are more likely to feel danger and threat, and easily to get nervous, feared, worried and anxious. These emotional responses can be reflected in the individuals' physiological, psychological and behavioral aspects.

\section{Conclusion}

By a comprehensive exploration of effects of stressor and neuroticism on stress response, the study obtained following conclusions:

- The interaction term of stressor (work) and neuroticism as well as two stressors (health and social) are predictors of psychological stress response;

- The interaction term of stressor (work) and neuroticism as well as three stressors (health, family and social) are predictors of physiological stress response;

- The interaction term of stressor (health) and neuroticism as well as two stressors (social and family) are predictors of behavioral stress response;

- The interaction term of stressor (health) and neuroticism, as well as three stressors (social, family and work) are predictors of total stress response.

\section{References}

Chu, X. Y., Fan, F. Q., Li, Y., Han, J., \& Han, K. (2015). Effects of Stressor and Personality Trait on Stress Response. Research in World Economy, 6(2), 1-11. http://dx.doi.org/10.5430/rwe.v6n2p

Chu, X. Y., Ma, Z. T., Li, Y., \& Han, J. (2015). Agreeableness, Extraversion, Stressor and Physiological Stress Response. International Journal of Social Science Studies, 3(4), 78-85. http://dx.doi.org/10.11114/ijsss.v3i4.857

Chu, X. Y., Ma, Z. T., Li, Y., \& Han, J. (2015). The Study of Relationship between Neuroticism, Stressor and Stress Response. Openness, Conscientiousness, Extraversion, Stressor and Psychological Stress Response. International Journal of Business Administration, 6(4), 1-8. http://dx.doi.org/10.5430/ijba.v6n4p

Costa, P. T. Jr., \& McCrae, R. R. (1980). Influence of extraversion and neuroticism on subjective well-being: happy and unhappy people. Journal of Personality and Social Psychology, 38, 668-678. http://dx.doi.org/10.1037/0022-3514.38.4.668

DeNeve, K. M., \& Cooper, H. (1998). The happy personality: A meta-analysis of 137 personality traits and

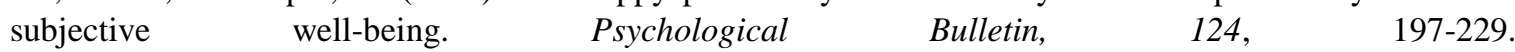
http://dx.doi.org/10.1037/0033-2909.124.2.197

Gross, J. J., Sutton, S. K., \& Ketelaar, T. (1998). Relations between Affect and Personality: Support for the Affect-Level and Affective-Reactivity Views. Personality and Social Psychology Bulletin, 24, 279-288. http://dx.doi.org/10.1177/0146167298243005

Han, J., Chu, X. Y., Song, H. C., \& Li, Y. (2014). Social Capital, Socioeconomic Status and Self-efficacy. Applied Economics and Finance, 1(2), 1-10. http://dx.doi.org/10.11114/aef.v2i1.607

Huang, M. E., \& Guo, D. J. (2003). Influence of extraversion and neuroticism on emotions. Psychological Science, 26(6), 1047-1051.

Lahey, B. B. (2009). Public health significance of neuroticism. American Psychologist, 64, 241-256. http://dx.doi.org/10.1037/a0015309

Meyer, G. J., \& Shack, J. R. (1989). The structural convergence of mood and personality: Evidence for old and new directions. Journal of Personality and Social Psychology, 57, 691-706. http://dx.doi.org/10.1037//0022-3514.57.4.691

Miller, D. J., Vachon, D. D., \& Lynam, D. R. (2009). Neuroticism,negative affect, and negative affect instability: Establishing convergent and discriminant validity using ecological momentary assessment. Personality and Individual Differences, 47, 873-877. http://dx.doi.org/10.1016/j.paid.2009.07.007

Muris, P., Roelofs, J., Rassin, E., Franken, I., \& Mayer, B. (2005). Mediating effects of rumination and worry on the links between neuroticism, anxiety and depression. Personality and Individual Differences, 39, 1105-1111. http://dx.doi.org/10.1016/j.paid.2005.04.005

$\mathrm{Ng}$, W. (2009). Clarifying the relation between neuroticism and positive emotions. Personality and Individual Differences, 47, 69-72. http://dx.doi.org/10.1016/j.paid.2009.01.049 
Ng, W., \& Diener, E. (2009). Personality differences in emotions: Does emotion regulation play a role? Journal of Individual Difference, 30, 100-106. http://dx.doi.org/10.1027/1614-0001.30.2.100

Osorio, L. C., Cohen, M., Escobar, S. E., Salkowski-Bartlett, A., \& Compton, R. J. (2003). Selective attention to stressful distracters: Effects of neuroticism and gender. Personality and Individual Differences, 34, 831-844. http://dx.doi.org/10.1016/s0191-8869(02)00074-0

Pervin, L. A. (2001). Science of personality (pp. 211-219). Shanghai: East China Normal University Press.

Robinson, M. D., Ode, S., Moeller, S. K., \& Goetz, P. W. (2007). Neuroticism and affective priming: Evidence for a neuroticism-linked negative schema. Personality and Individual Differences, 42, 1221-1231. http://dx.doi.org/10.1016/j.paid.2006.09.027

Verbeke, W., \& Kenhove, P. V. (2002). Impact of emotional stability and attitude on consumption decisions under risk: the Coca-Cola crisis in Belgium. Journal of Health Communication, 7, 445-472. http://dx.doi.org/10.1080/10810730290001819

Watson, D., \& Clark, L. A. (1992). On traits and temperament: General and specific factors of emotional experience and their relation to the five factor model. Journal of Personality, 60, 441-476. http://dx.doi.org/10.1111/j.1467-6494.1992.tb00980.x

Weiss, A., \& Costa, P. T. (2005). Domain and facet personality predictors of all-cause mortality among Medicare $\begin{array}{lllllll}\text { patients aged } 65 \text { to } 100 . & \text { Psychosomatic Medicine, 67, } & \text { 724-733. }\end{array}$ http://dx.doi.org/10.1097/01.psy.0000181272.58103.18

\section{Copyrights}

Copyright for this article is retained by the author(s), with first publication rights granted to the journal.

This is an open-access article distributed under the terms and conditions of the Creative Commons Attribution license (http://creativecommons.org/licenses/by/3.0/). 